\title{
Rapid Palatal Expansion Should Not Be Trivialized: Two Case Reports of Unexpected Complications
}

\author{
Gaia Lopponi ${ }^{1,2}$ Bortolo Giuliano Maino ${ }^{3,4}$ Domenico Dalessandri5,๑
}

\author{
${ }^{1}$ Department of Endodontics, University of Florence, Firenze, Italy \\ ${ }^{2}$ Department of Orthodontics, University of Cagliari, Cagliari, Italy \\ ${ }^{3}$ Postgraduate Orthodontic School, Ferrara University, Ferrara, Italy \\ 4Private Practice, Vicenza, Italy \\ ${ }^{5}$ Department of Medical and Surgical Specialties, Radiological \\ Sciences, and Public Health, School of Dentistry, University of \\ Brescia, Brescia, Italy
}

\author{
Address for correspondence Gaia Lopponi, DMD, MS, Department \\ of Endodontics, University of Florence, Piazza San Marco 4, \\ 50121 Firenze, Italy (e-mail: gaia.lopponi@unifi.it).
}

\begin{abstract}
Keywords

- rapid maxillary expansion

- orthodontic

- orthopaedic

- pediatric

- complications

Rapid maxillary expansion is one of the most common orthodontic procedures performed in the pediatric population, easy to accomplish and compliance free, therefore really effective. Nevertheless, a careful diagnosis and frequent clinical follow-up appointments are necessary during the first weeks of active treatment. The purpose of this article was to report two cases of unexpected complications during orthopaedic maxillary expansion, involving two 7-year-old female patients, one presenting a saddle nose with suborbital hematoma and the other one an asymmetric palatal expansion. In both cases, the clinical situation progressively relapsed during the months following expansion appliance removal.
\end{abstract}

\section{Introduction}

Rapid maxillary expansion (RME) is one of the most common orthodontic procedures. The aim of RME is to orthopaedically expand the maxillary dental arch and palate through distraction of the mid-palatal suture. This procedure is used to resolve different malocclusions, such as transverse discrepancy (TD), posterior crossbite (PC), and dental crowding. Furthermore, it is also used to expand upper airways in patients with obstructive respiratory disorders. ${ }^{1-7}$

The purpose of this article is to show two unexpected side effects affecting the facial skeleton that arose as a consequence of the application of a RME procedure.

\section{Case Reports}

The first patient, a 7-year-old female in her early mixed dentition stage, presented with a single tooth crossbite of

\section{published online} June 25, 2021
DOI https://doi.org/

$10.1055 / \mathrm{s}-0041-1728840$

ISSN 1305-7456 the permanent maxillary left lateral incisor and a complete right PC, from the permanent maxillary right lateral incisor to the permanent maxillary right first molar, with mandibular shift in habitual occlusion. In centric relation, the diagnosis was a bilateral PC with a transverse deficiency of the upper jaw measuring $7 \mathrm{~mm}$ : she was then scheduled for a RME with a Hyrax device-simpler to build, easier to clean for the patient, and causing less interference with the patient's speech-with bands on the primary maxillary second molars (-Fig. 1). ${ }^{8,9}$ A classic activation protocol, with two quarter turns per day until TD overcorrection had been achieved, was chosen. ${ }^{10,11}$ The child's mother was instructed on how to properly activate the device. At the follow-up visit after 14 days (seven complete rounds, that is to say, 28 quarter turns), despite the correctness of the procedure used, the patient presented with a nasal anatomy alteration consisting in a saddle nose and a suborbital hematoma ( - Fig. 2). According to the mother, these presented during the previous night after
(C) 2021. European Journal of Dentistry.

This is an open access article published by Thieme under the terms of the Creative Commons Attribution-NonDerivative-NonCommercial-License, permitting copying and reproduction so long as the original work is given appropriate credit. Contents may not be used for commercial purposes, or adapted, remixed, transformed or built upon. (https://creativecommons.org/licenses/by-nc-nd/4.0/).

Thieme Medical and Scientific Publishers Pvt. Ltd. A-12, 2nd Floor, Sector 2, Noida-201301 UP, India 

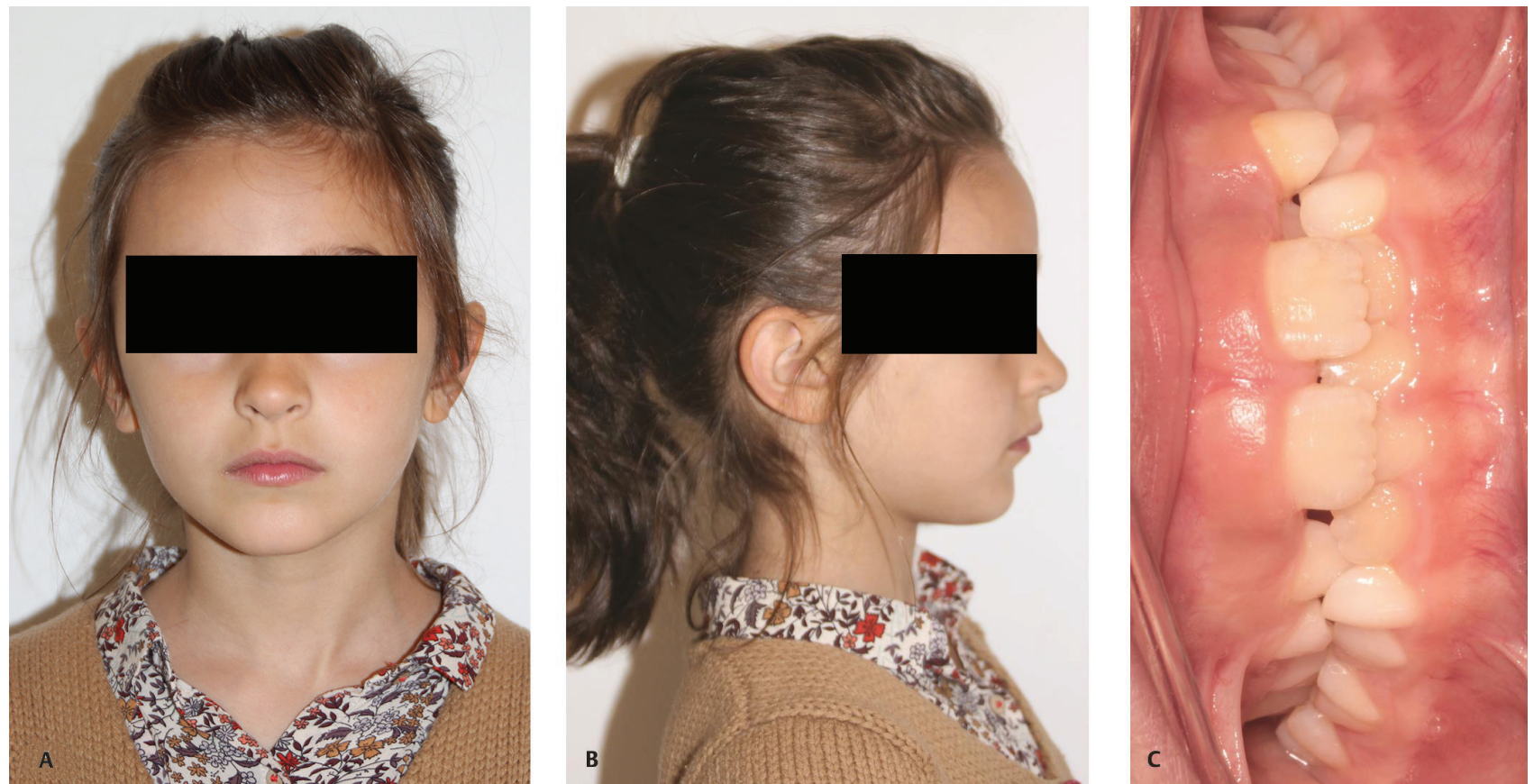

Fig. 1 The first patient, a 7-year-old female in her early mixed dentition stage, before treatment. (A) Facial frontal view. (B) Facial lateral view. (C) Intraoral frontal view, showing the right crossbite in maximum intercuspation position.
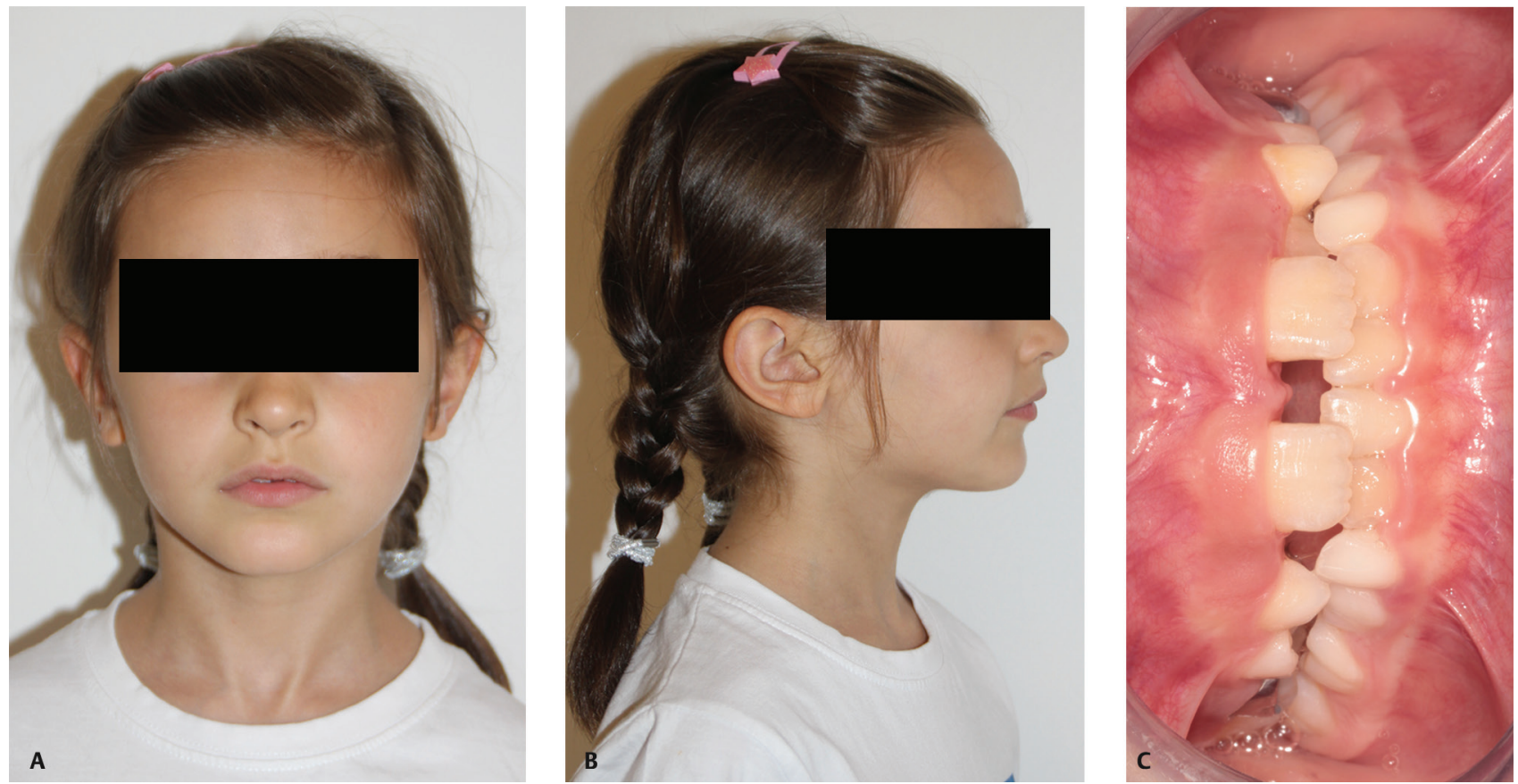

Fig. 2 The first patient after orthopaedic maxillary expansion with a Hyrax device with bands on the primary maxillary second molars. (A) Facial frontal view. (B) Facial lateral view. (C) Intraoral frontal view, showing the interpermanent maxillary central incisors diastema opening; (D) intraoral upper occlusal view.

the last activation, accompanied by more pain in the nasal area than there was after previous activations. As shown by the width of her interpermanent maxillary central incisors diastema, the patient had an important orthopaedic expansion of her jawbone. This type of expansion normally occurs in a triangular manner, with its base at the level of the nasal cavities and its apex at the level of the palatine processes. ${ }^{12}$
Three-quarters of the floor of the nasal cavities are composed anteriorly by the palatine process of the jawbone, with the posterior third composed by the horizontal lamina of the palatine bone. In this patient, in whom there was a large component of sutural distraction and a small dental component, the procedure caused a collapse and subluxation of the foot of the septal cartilage, manifesting itself at the level of the 
mucosa in the area where the nasal bone and cartilage are joined. The appliance was then blocked and, after 3 months, relapse was completed ( $\boldsymbol{- \text { Fig. }}$ 3). No substantial changes were noticed in the following 12 months.

The second patient, a 7-year-old hyperdivergent female presenting a maxillary arch constriction in association with a dentoalveolar discrepancy, was scheduled for a RME with a CAD/CAM Haas expander-where the arms that link the central expansion screw to the dental elements are embedded in an acrylic resin plate that rests on the mucosa of the palatal vault to obtain combined palatal and dental supportsbonded on primary maxillary second molars and canines ( Fig. 4). ${ }^{13}$ Screw activation protocol was two quarter turns $(0.4 \mathrm{~mm})$ per day for 20 days, to obtain $8 \mathrm{~mm}$ of screw expansion. After 10 days, the expansion was symmetric and there was a diastema between the permanent maxillary central incisors. After 15 days the patient was seen in emergency: the mother referred that the previous night the patient woke up presenting hemifacial pain and, during the following hours, a progressively increasing unilateral expansion of the right maxillary process. At clinical and radiological examination a suture opening was demonstrated, with an unilateral expansion and downward movement of the right hemimaxillary process: the resistance of the circummaxillary sutures was probably not equivalent on both sides, and therefore, after the sudden loosening of the sutures on the right side, there was a release of elastic energy from the left side with unilateral expansion toward the right side ( Fig. 5)..$^{14,15}$ The appliance was then removed just after the appearance of the side effects and, after 3 months, relapse was completed (-Fig. 6). No substantial changes or adverse events were noticed during the following year.

\section{Discussion}

Orthodontic appliances used to achieve a maxillary expansion can be divided, according to the anatomical structure where the orthopaedic force is applied, in tooth-borne, bone-borne, and hybrid. In tooth-borne appliances the expansion force is applied to teeth and then transferred to the maxillary bone, while in bone-borne appliances the force is applied to the bone by means of orthodontic mini-implants (OMIs). In hybrid appliances, both teeth and OMI are used to anchor the palatal expander.

The action of the tooth-borne rapid maxillary expander is based on the application of heavy and continuous forces to exceed the limit required for orthodontic movement, producing hyalinization by compression of the periodontal ligament, pseudoankylosis of the supporting elements, and consequently the opening of the median palatine suture, confirmed by the opening of an interpermanent maxillary central incisors diastema. ${ }^{16,17}$ This interdental space resolves spontaneously thanks to the traction of the interdental fibers that bring the crowns together during the first month and later, in approximately 4 months, the roots. In the meantime, osteogenesis fills in the sutural distraction created..$^{18}$ Some side effects associated with this expansion protocol are: a temporary reduction of the bite force, impossibility to simultaneously correct molar rotation, appliance spontaneous debonding, and periodontal breakage. ${ }^{19-22}$

We opted for a tooth-borne device in both patients presented in this article. We bonded a Hyrax expander in the first patient and a Haas-type expander in the second, both anchored to the second upper deciduous molars by means of orthodontic bands. No elastics were used in these two
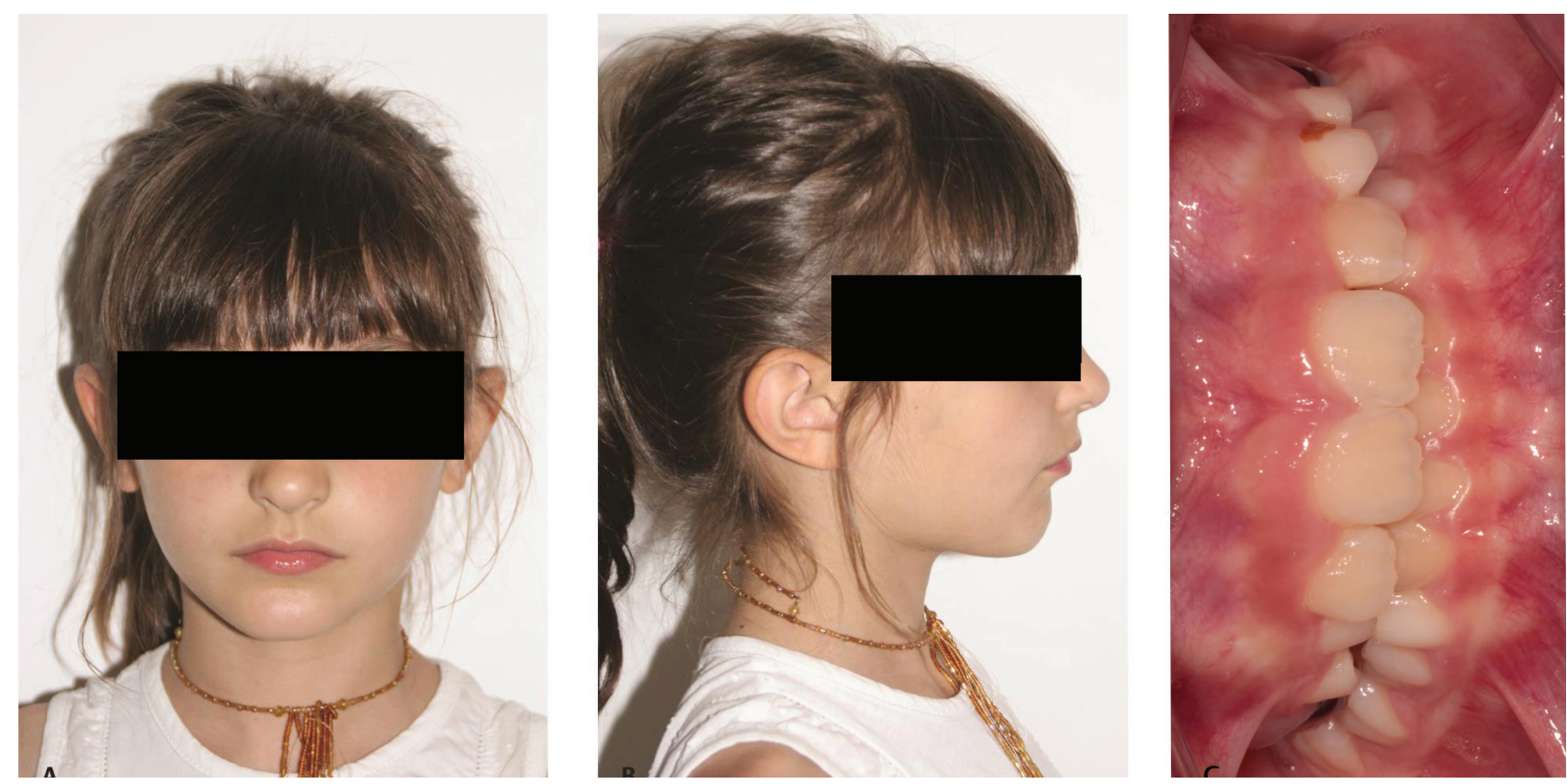

Fig. 3 The first patient 3 months after active expansion. (A) Facial frontal view. (B) Facial lateral view with restored nasal anatomy. (C) Intraoral frontal view showing diastema closing and persistent posterior crossbite (PC). 

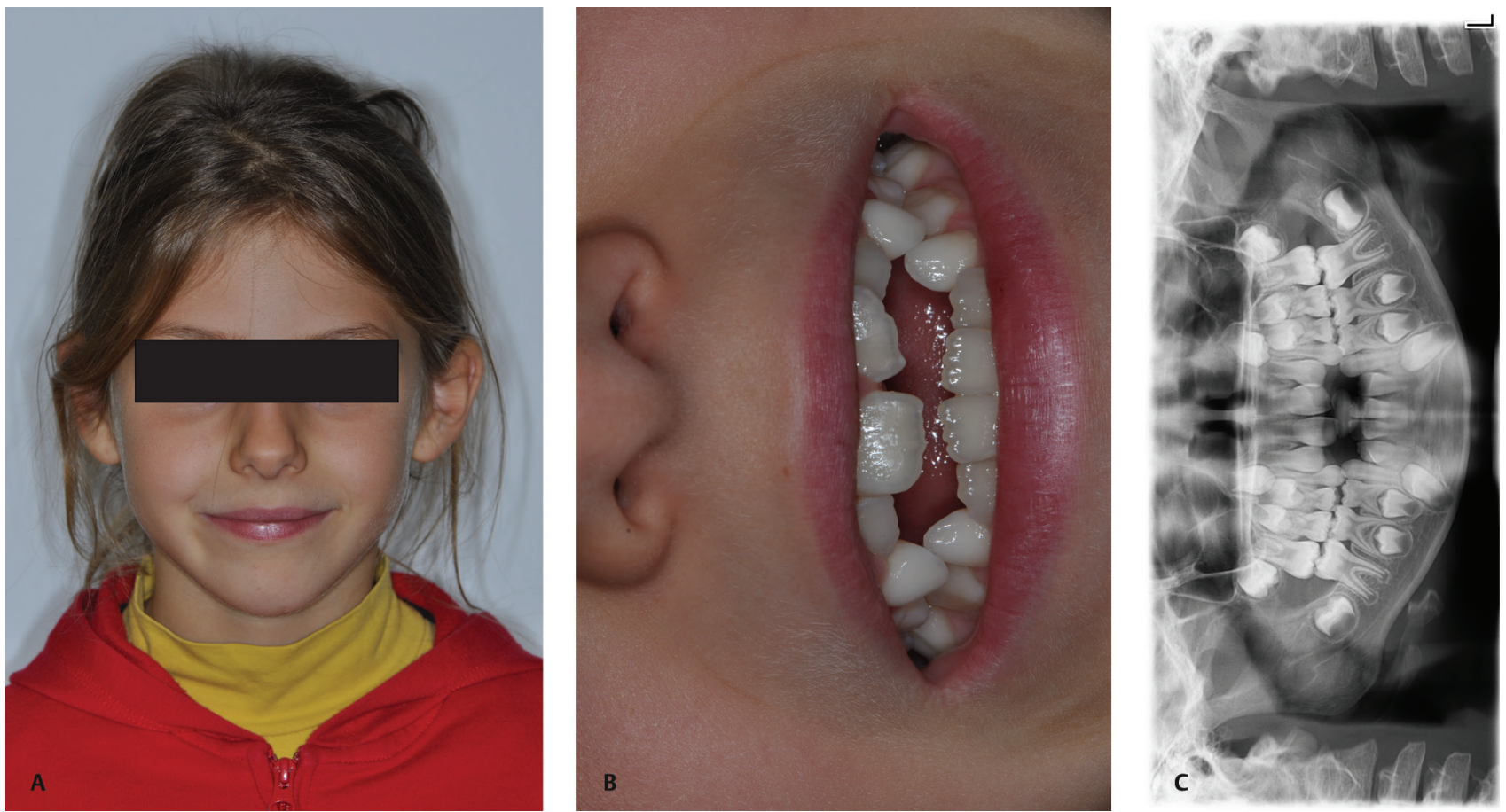

Fig. 4 The second patient, a 7-year-old female hyperdivergent female presenting a maxillary arch constriction in association with a dentoalveolar discrepancy, before treatment. (A) Facial frontal view. (B) Smile frontal view. (C) Orthopantomography.
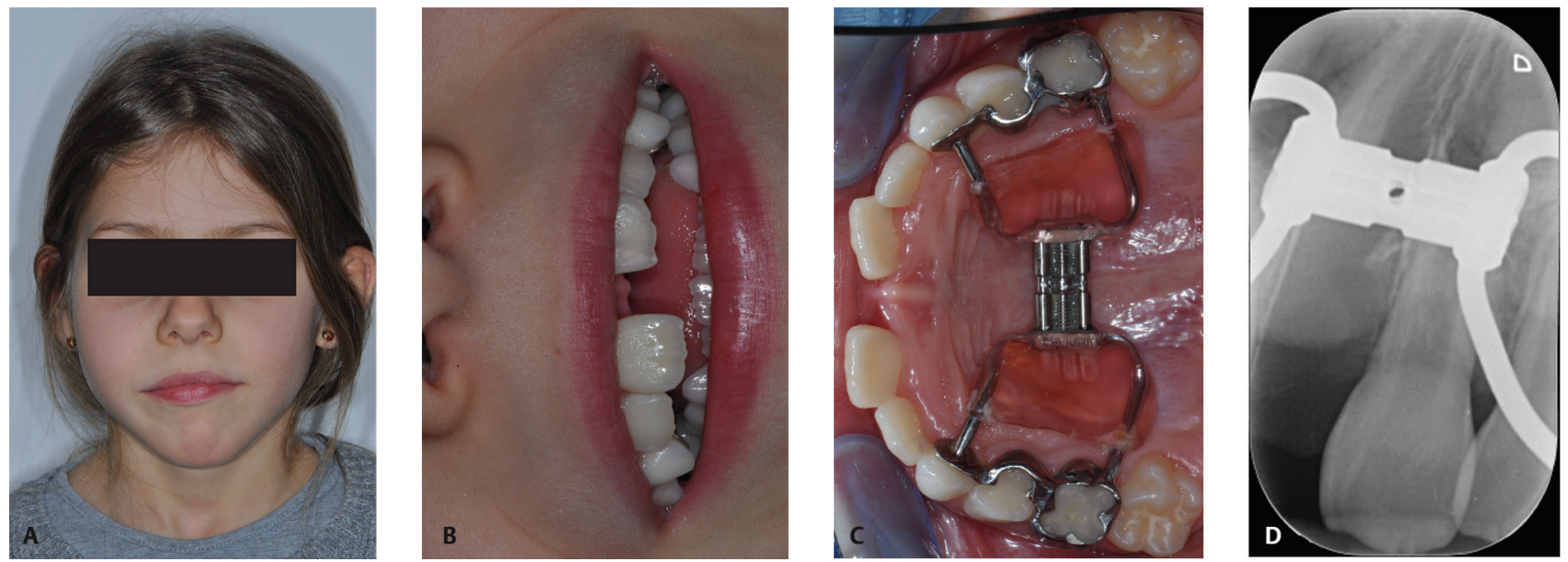

Fig. 5 The second patient after orthopaedic maxillary expansion with a CAD/CAM Haas expander bonded on primary maxillary second molars and canines. (A) Facial frontal view. (B) Smile frontal view. (C) Intraoral upper occlusal view. (D) Periapical X-ray demonstrating asymmetric suture opening.

patients. We followed a rapid palatal expansion protocol for both patients with a daily activation of $0.40 \mathrm{~mm}$ until correction of the TD, when the palatal cusp of the first permanent molar is in contact with the buccal cusp of the first permanent lower molar, even if it caused having the deciduous molars in the scissor bite position. Both patients showed side effects between the 13th and the 14th day of the activation procedure: the active phase was immediately stopped and a spontaneous remission was observed after 3 months. No functional impairment of the temporomandibular joint, investigated through clinical examination, occurred during device activation and up to 1 year after treatment.
Before treatment, the two patients had different malocclusions: the first patient presented a unilateral crossbite with a functional shift and no dentoalveolar discrepancy; the second patient presented a massive crowding in the frontal area of the upper arch and an anterior open bite. But both patients showed an asymmetrical pattern, which could have played a role in the uncontrolled effect of the orthopaedic forces applied to teeth.

A therapeutic alternative in to tooth-borne expanders are hybrid or bone-borne expanders, based on the insertion of OMI. Their use to produce palatal expansion was developed for the first time in 1999 by Mommaerts to avoid some 

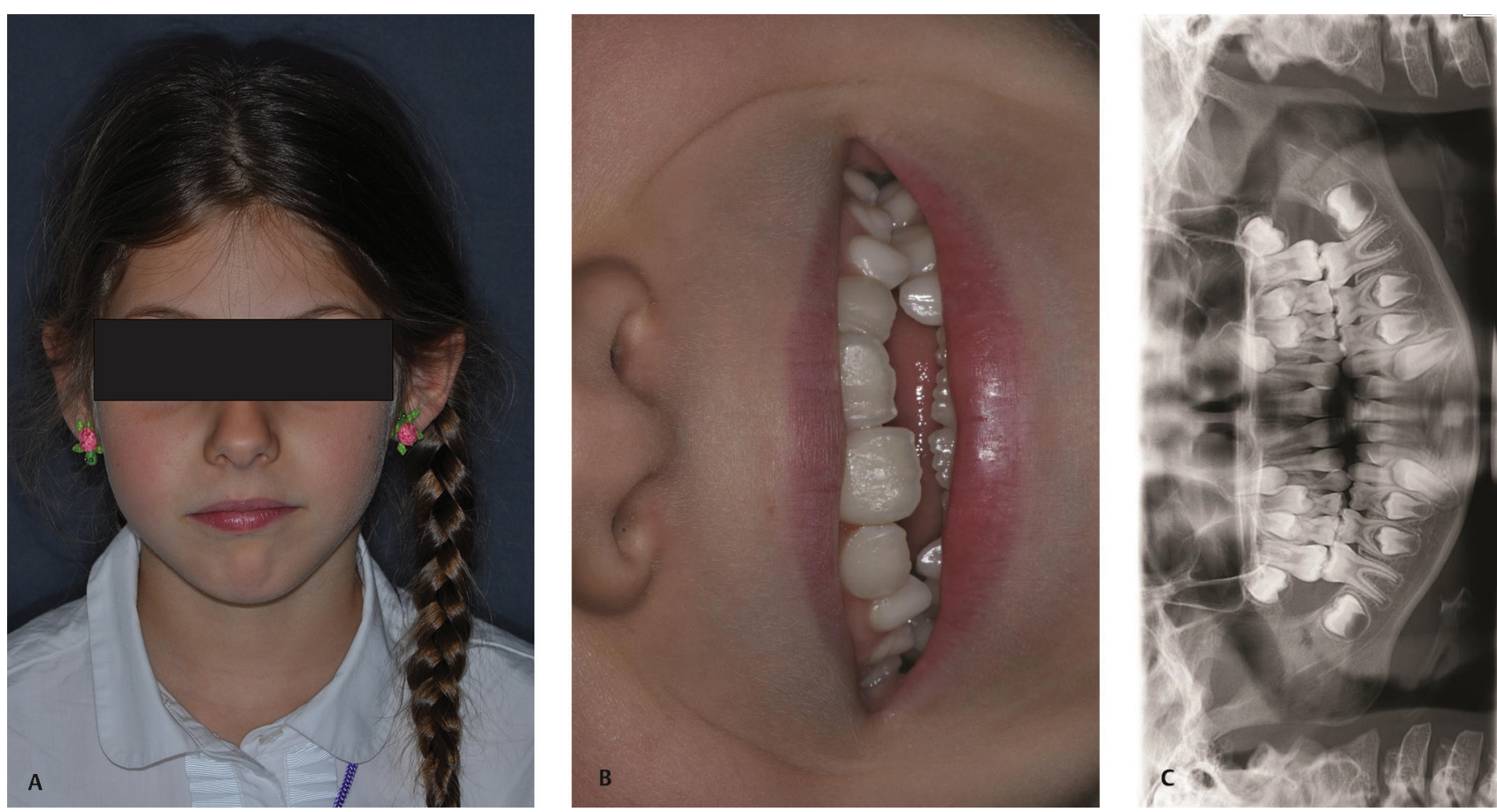

Fig. 6 The second patient 3 months after expander removal. (A) Facial frontal view. (B) Smile frontal view. (C) Orthopantomography.

common side effects like the movement and the vestibuloversion of molars. ${ }^{23}$ Actually, even using deciduous molars as anchorage it is possible to avoid unwanted vestibular inclination of first permanent molars, but sometimes they are not available or they cannot support the expansion forces of the palatal expander. The choice of using OMI or teeth-borne appliances to achieve palatal expansion depends on the patient's periodontal status, the quality of the available dental elements, the patient's skeletal age, and the need to have a greater skeletal expansion with a low amount of dental tipping. ${ }^{24}$ The difference in pain perception with the same screw activation protocol of two quarter per day between teeth-borne appliance and bone-borne appliance is not clinically significant. ${ }^{25,26}$ Regarding the quantity and quality of maxillary expansion some authors found that skeletal OMI devices are better and more effective, while others found no differences. In any case, skeletal devices were found to present a better hygiene control and less premolar tipping. 27-30

Anyway, palatal expansion acts expressly at the level of the median palatine suture. However, given that the maxillary bones are in a central position with respect to the splanchnocranium and that they articulate with several bones of the facial skeleton, while contributing to the formation of the oral, orbital, and nasal cavities, palatal expansion also affects the circummaxillary structures and sutures and moves the maxilla downward and forward. ${ }^{31-33}$ In addition, palatal expansion also influences the auditory region of the skull through its connection from the tympanic cavity to the nasopharynx created by the Eustachian tubes. ${ }^{34}$ Timms demonstrated how RME can modify the soft tissue of the palate and the pharynx and improve Eustachian tube drainage, which results in a decrease in middle ear infections and hearing loss. ${ }^{35}$ Though still controversial, it has been shown that there is an association between oral respiration and reduced air cavity volume and that the growth of the maxillary complex and the upper airways are related to one another. ${ }^{36}$ In particular, how the disjunction of the maxillary bones influences the separation of the nasal walls and creates a lowering of the palatal vault, on which the cartilaginous foot of the septum rests centrally, has also been demonstrated. ${ }^{37}$ This can produce both positive effects, such as increased permeability of the upper airway and stretching of the septum cartilage with decreased nasal septum deviation as well as side effects such as hematomas and saddle nose. While the first is still controversial whether it has a long-term effect, in the cases we have considered here, the latter had a spontaneous remission within a month after the active treatment phase was stopped ${ }^{38}$ Referring to this, recent studies have showed that the stress distribution around the circumaxillary sutures is reduced in micro-implants assisted rapid palatal expansion. ${ }^{39,40}$ Nevertheless, the risk of asymmetric expansion is still present, and in a study published by Kim et al, it was found to be $30.3 \%$, without predictive factors, even if it was more frequent in patients with chin deviation. ${ }^{41}$

It follows that, regardless of the subject's chronological age, RME is a therapy in which the distraction of the mid-palatal suture is dependent on the patient's skeletal maturity and sutural density, therefore potentially presenting significant undesirable effects if not strictly monitored: RME appliances should always be applied only after a correct diagnosis and a strict follow-up protocol during active expansion should be scheduled. ${ }^{42}$ 


\section{Authors' Contributions}

The responsibility of G.L. was to conceive and write the original draft. The responsibility of G.B.M. was to supervise and review the manuscript. The responsibility of D.D. was to write the original draft and synthesize data.

\section{Conflict of Interest}

None declared.

\section{References}

1 Seif-Eldin NF, Elkordy SA, Fayed MS, Elbeialy AR, Eid FH. Transverse skeletal effects of rapid maxillary expansion in pre and post pubertal subjects: a systematic review. Open Access Maced J Med Sci 2019;7(3):467-477

2 Mutinelli S, Manfredi M, Guiducci A, Denotti G, Cozzani M. Anchorage onto deciduous teeth: effectiveness of early rapid maxillary expansion in increasing dental arch dimension and improving anterior crowding. Prog Orthod 2015;16:22

3 Mutinelli S, Cozzani M, Manfredi M, Bee M, Siciliani G. Dental arch changes following rapid maxillary expansion. Eur J Orthod 2008;30(5):469-476

4 Dalessandri D, Tonni I, Dianiskova S, et al. Rapid palatal expander vs. quad-helix in the orthodontic treatment of cleft lip and palate patients. Minerva Stomatol 2016;65(2):97-103

5 Zeng J, Gao X. A prospective CBCT study of upper airway changes after rapid maxillary expansion. Int J Pediatr Otorhinolaryngol 2013;77(11):1805-1810

6 Petrén S, Bondemark L, Söderfeldt B. A systematic review concerning early orthodontic treatment of unilateral posterior crossbite. Angle Orthod 2003;73(5):588-596

7 Oliva G, Huanca Ghislanzoni L, Dalessandri D, Silvestrini-Biavati A, Ugolini A. Palatal changes in crossbite patients treated with rapid maxillary expansion vs untreated ones: a geometric morphometric study. Orthod Craniofac Res 2020;23(4):439-444

8 Cozzani M, Guiducci A, Mirenghi S, Mutinelli S, Siciliani G. Arch width changes with a rapid maxillary expansion appliance anchored to the primary teeth. Angle Orthod 2007;77(2): 296-302

9 Cozzani M, Rosa M, Cozzani P, Siciliani G. Deciduous dentition-anchored rapid maxillary expansion in crossbite and non-crossbite mixed dentition patients: reaction of the permanent first molar. Prog Orthod 2003;4:15-22

10 Baldini A, Nota A, Santariello C, Assi V, Ballanti F, Cozza P. A comparative assessment of changes in dental arches associated with different activation protocols of rapid maxillary expansion. Eur J Paediatr Dent 2018;19(1):35-39

11 Baldini A, Nota A, Santariello C, et al. Sagittal dentoskeletal modifications associated with different activation protocols of rapid maxillary expansion. Eur J Paediatr Dent 2018; 19(2):151-155

12 Haas AJ. Rapid expansion of the maxillary dental arch and nasal cavity by opening the midpalatal suture. Angle Orthod 1961;31(2):73-90

13 Graf S, Cornelis MA, Hauber Gameiro G, Cattaneo PM. Computer-aided design and manufacture of hyrax devices: can we really go digital? Am J Orthod Dentofacial Orthop 2017;152(6):870-874

14 Savoldi F, Tsoi JKH, Paganelli C, Matinlinna JP. Biomechanical behaviour of craniofacial sutures during distraction: an evaluation all over the entire craniofacial skeleton. Dent Mater 2017;33(7):e290-e300

15 Savoldi F, Tsoi JKH, Paganelli C, Matinlinna JP. Evaluation of rapid maxillary expansion through acoustic emission technique and relative soft tissue attenuation. J Mech Behav Biomed Mater 2017;65:513-521
16 Agarwal A, Mathur R. Maxillary expansion. Int J Clin Pediatr Dent 2010;3(3):139-146

17 Arvind Kumar S, Muruganandham GD, Sharma S. Rapid maxillary expansion: a unique treatment modality in dentistry. J Clin Diagn Res 2011;5(4):906-911

18 Uysal T, Amasyali M, Enhos S, Sonmez MF, Sagdic D. Effect of ED-71, a new active vitamin D analog, on bone formation in an orthopedically expanded suture in rats. A histomorphometric study. Eur J Dent 2009;3(3):165-172

19 Uzuner FD, Odabasi H, Acar S, Tortop T, Darendeliler N. Evaluation of the effects of modified bonded rapid maxillary expansion on occlusal force distribution: a pilot study. EurJ Dent 2016;10(1):103-108

20 Tonni I, Iannazzi A, Piancino MG, Costantinides F, Dalessandri D, Paganelli C. Asymmetric molars' mesial rotation and mesialization in unilateral functional posterior crossbite and implications for interceptive treatment in the mixed dentition. Eur J Orthod 2017;39(4):433-439

21 Ozzeybek Can FS, Turkkahraman H. Effects of rapid maxillary expansion and facemask therapy on the soft tissue profiles of class III patients at different growth stages. Eur J Dent 2019; 13(2):143-149

22 Matarese G, Isola G, Ramaglia L, et al. Periodontal biotype: characteristic, prevalence and dimensions related to dental malocclusion. Minerva Stomatol 2016;65(4):231-238

23 Mommaerts MY. Transpalatal distraction as a method of maxillary expansion. Br J Oral Maxillofac Surg 1999;37(4):268-272

24 Copello FM, Marañón-Vásquez GA, Brunetto DP, et al. Is the buccal alveolar bone less affected by mini-implant assisted rapid palatal expansion than by conventional rapid palatal expansion? A systematic review and meta-analysis. Orthod Craniofac Res 2020;23(3):237-249

25 Migliorati M, Drago S, Gallo F, et al. Immediate versus delayed loading: comparison of primary stability loss after miniscrew placement in orthodontic patients-a single-centre blinded randomized clinical trial. Eur J Orthod 2016;38(6):652-659

26 Prado J, Pardo H, Bravo M. Rapid maxillary expansion assisted by mini-implants anchorage: a case report. Int Orthod 2019;17(1):159-169

27 Khosravi M, Ugolini A, Miresmaeili A, et al. Tooth-borne versus bone-borne rapid maxillary expansion for transverse maxillary deficiency: a systematic review. Int Orthod 2019;17(3): 425-436

28 Baldini A, Nota A, Santariello C, Assi V, Ballanti F, Cozza P. Influence of activation protocol on perceived pain during rapid maxillary expansion. Angle Orthod 2015;85(6):1015-1020

29 Lagravère MO, Carey J, Heo G, Toogood RW, Major PW. Transverse, vertical, and anteroposterior changes from bone-anchored maxillary expansion vs traditional rapid maxillary expansion: a randomized clinical trial. Am J Orthod Dentofacial Orthop 2010;137(3):304.e1-304.e12, discussion 304-305

30 Lagravère MO, Gamble J, Major PW, Heo G. Transverse dental changes after tooth-borne and bone-borne maxillary expansion. Int Orthod 2013;11(1):21-34

31 Leonardi R, Sicurezza E, Cutrera A, Barbato E. Early post-treatment changes of circumaxillary sutures in young patients treated with rapid maxillary expansion. Angle Orthod 2011; 81(1):36-41

32 Haas AJ. Palatal expansion: just the beginning of dentofacial orthopedics. Am J Orthod 1970;57(3):219-255

33 da Silva Filho OG, Boas MC, Capelozza Filho L. Rapid maxillary expansion in the primary and mixed dentitions: a cephalometric evaluation. Am J Orthod Dentofacial Orthop 1991; 100(2):171-179

34 Taşpinar F, Uçüncü $\mathrm{H}$, Bishara SE. Rapid maxillary expansion and conductive hearing loss. Angle Orthod 2003;73(6):669-673 
35 Timms DJ. A study of basal movement with rapid maxillary expansion. Am J Orthod 1980;77(5):500-507

36 Enlow DH, Facial Growth. 3rd ed. Philadelphia: WB Saunders Co; 1990

37 Buck LM, Dalci O, Darendeliler MA, Papageorgiou SN, Papadopoulou AK. Volumetric upper airway changes after rapid maxillary expansion: a systematic review and meta-analysis. Eur J Orthod 2017;39(5):463-473

38 Gokce G, Veli I, Yuce YK, Isler Y.. Efficiency evaluation of rapid maxillary expansion treatment on nasal septal deviation using tortuosity ratio from cone-beam computer tomography images. Comput Methods Programs Biomed 2020;188-105260

39 MacGinnis M, Chu H, Youssef G, Wu KW, Machado AW, Moon W. The effects of micro-implant assisted rapid palatal expansion (MARPE) on the nasomaxillary complex-a finite element method (FEM) analysis. Prog Orthod 2014;15(1):52

40 Kunz F, Linz C, Baunach G, Böhm H, Meyer-Marcotty P. Expansion patterns in surgically assisted rapid maxillary expansion : transpalatal distractor versus hyrax appliance. J Orofac Orthop 2016;77(5):357-365

$41 \mathrm{Kim} \mathrm{KA}$, Oh SH, Kim BH, Kim SJ. Asymmetric nasomaxillary expansion induced by tooth-bone-borne expander producing differential craniofacial changes. Orthod Craniofac Res 2019;22(4):296-303

42 Angelieri F, Franchi L, Cevidanes LH. Bueno-Silva B, McNamara JA Jr. Prediction of rapid maxillary expansion by assessing the maturation of the midpalatal suture on cone beam CT. Dental Press J Orthod 2016;21(6):115-125 